Check for updates

\title{
Osteolysis In a Well Fixed Acetabular Cup, Retain or Revise?
}

\author{
Mojieb Manzary, MD, CM, FRCSC ${ }^{1}$
}

\begin{abstract}
Wear and Osteolysis are the commonest cause of aseptic loosening in Total Hip Arthroplasty (THA), requiring revision. A less invasive approach could be undertaken in terms of an earlier intervention by isolated the liner change only. Indications and contraindications of each approach are reviewed with some technical tips. Outcome results have shown that isolated liner exchange alone does have a higher risk of instability.
\end{abstract}

\section{Introduction}

There is no doubt that THA has been a very successful procedure in terms of pain relief and improving the overall quality of life of those who receive it. However, it does have a limitation in terms of long-term survivorship which leads to patients undergoing one or more THA revisions in their lifetime.

Osteolysis is the commonest cause of aseptic loosening in contemporary THA. It could be defined as the process of progressive destruction of periprosthetic bone, characterized on serial radiographs as radiolucent lines and/or cavitation at the bone implant or cement bone interface (Figure 1). $[4,13,17]$

Although traditionally it was called the Cement Disease, it has been agreed that other particles, e.g, metal

1 Adult Joint Reconstructive Surgery

Head, Orthopedic Services

John Hopkins Aramco Health Care

Dhahran

Saudi Arabia

(C) 2015 Mojieb Manzary. All rights reserved

DOI: 10.15438/rr.4.4.86 • ISSN 2331-2262 (print) • ISSN 2331-2270 (online)

For complete copyright and licensing information please refer to the end of this article.

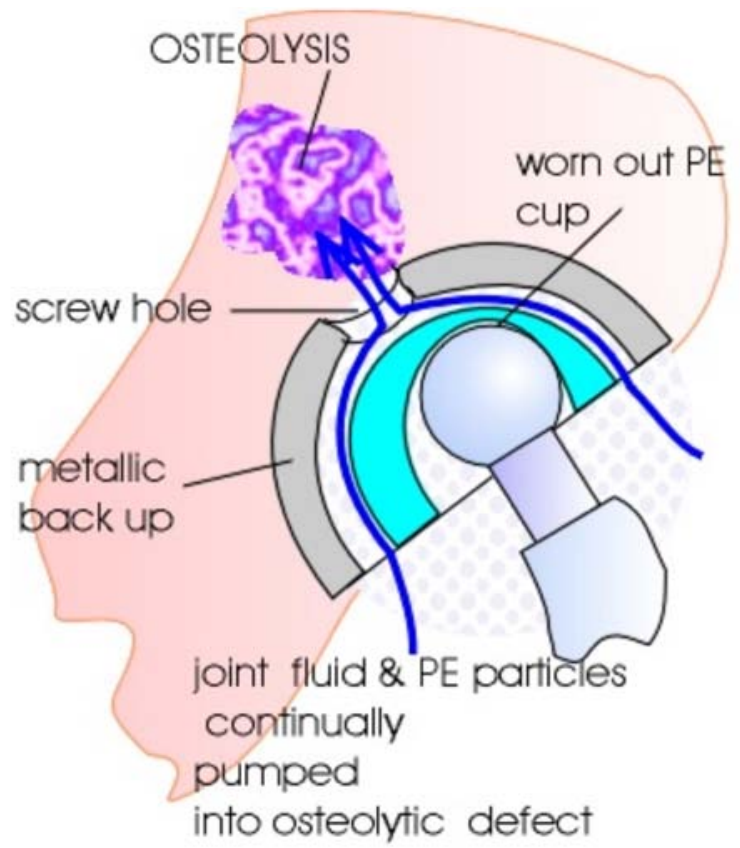

Figure 1. OSTEOLYSIS

debris, polyethylene wear particles, and bone fragments, are equally active in generating bone resorbing materials through an inflammatory process. $[13,17]$ 


\section{Discussion}

The prevalence of Osteolysis has varied over the years, in the early 1990's, with the ultramolecular high weight polyethylene (UMHWPE) sterilized in gamma irradiation in air, being the dominant poly liner used in THA, the rate of wear and Osteolysis did range from 10\%-70\%. [9]

The degree of symptomatology varies from being com-
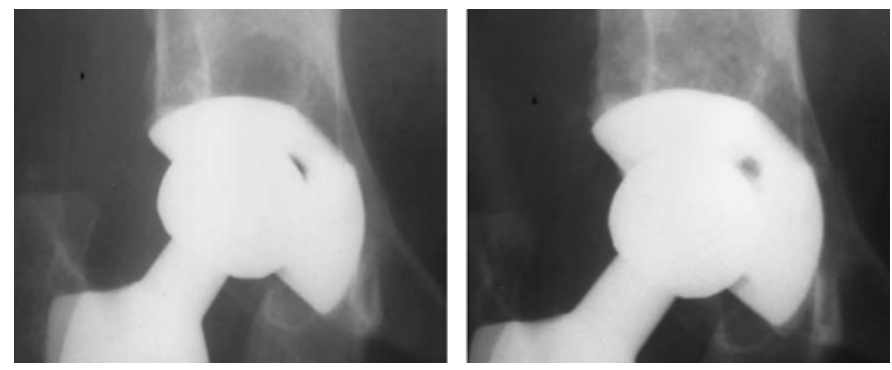

Figure 2. Osteolysis seen on xray

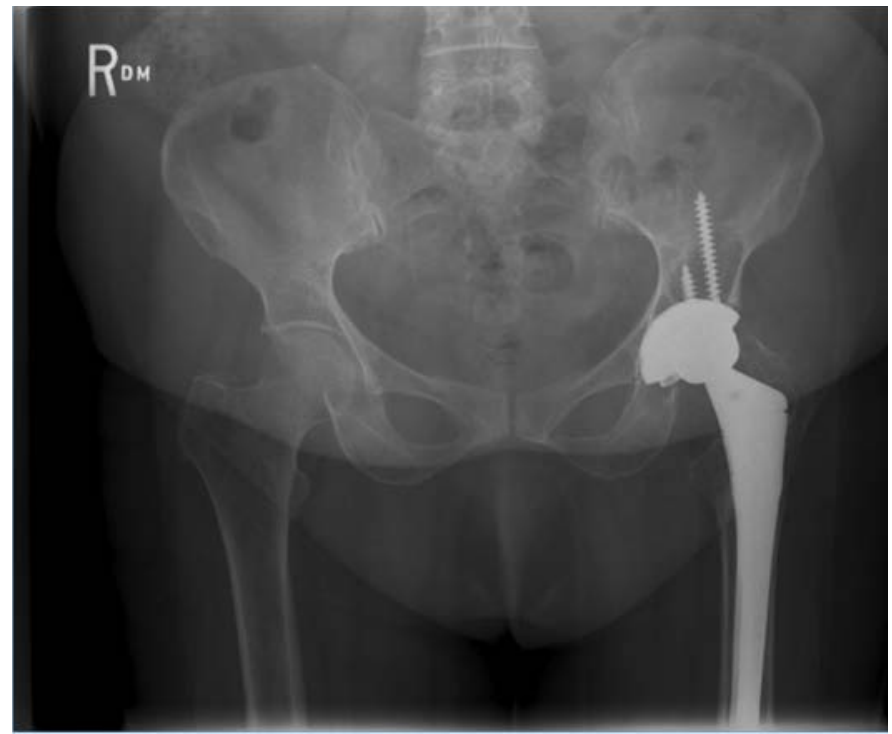

Figure 3. Osteolysis in an uncemented THA

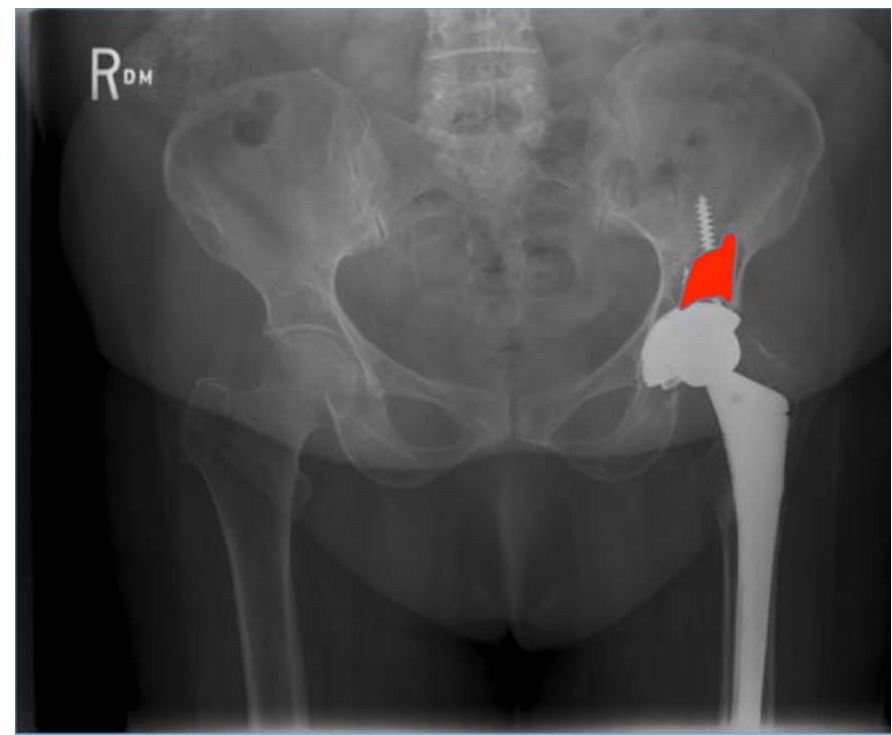

Figure 4. Osteolysis in Uncemented THA

Note the superoacetabular Area

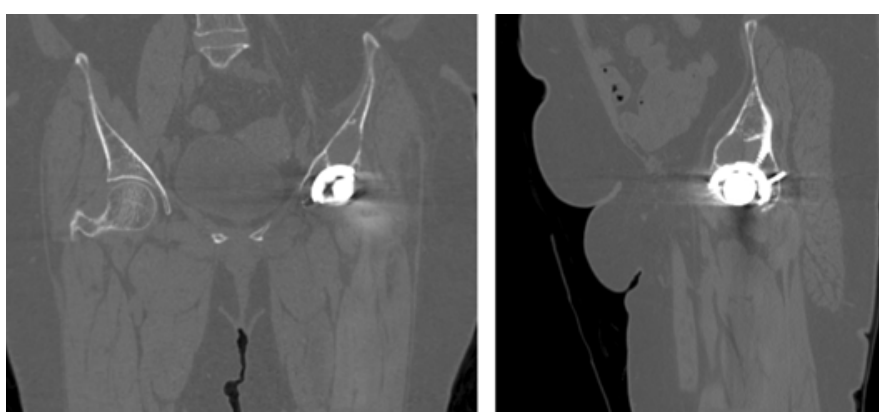

Figure 5. CT scan for Osteolysis
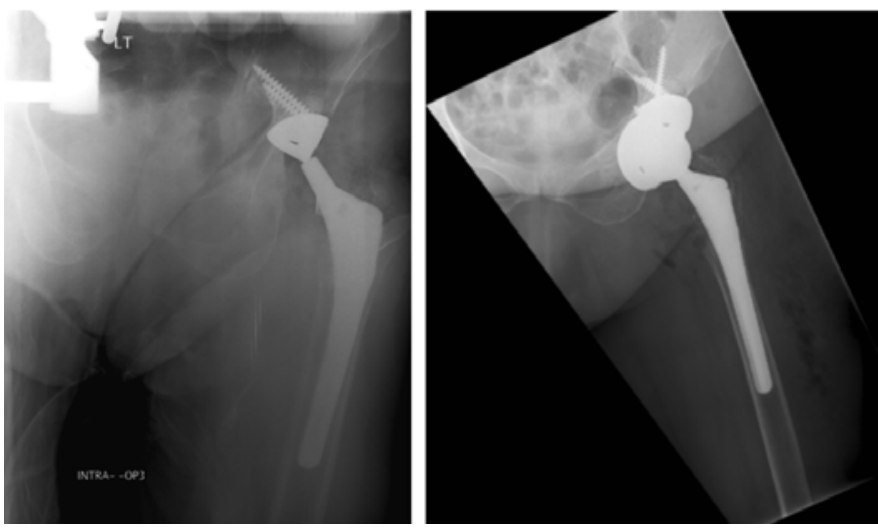

Figure 6. Osteolysis, defect management

pletely asymptomatic to catastrophic failure with periprosthetic fractures with or without instability. The task of the arthroplasty surgeon here lies in identifying the osteolytic process and developing a strategy of when and how to face the osteolytic challenge. In other words, having the right choice(s) to deal with this problem.

The pattern of osteolysis differs between cemented and cementless acetabular cups. $[10,18]$ In the cemented cup it's usually a linear pattern that has been described, whereas in the cups that have been inserted without cement it's an expansile pattern. [10,18] (Figures 2, 3, 4, 6)

A layer of fibrous tissue usually gets formed around the cemented acetabular component that may provide a path of least resistance along the planes of the layer, leading to a linear pattern rather than an expansile pattern. There is also a halo of sclerotic bone that usually develops peripheral to a component encapsulated by fibrous tissue.

This, along with the fibrous layer, may serve as a protective barrier to the expansion of the osteolysis to the more deeper, weaker, and porous cancellous layers of the acetabulum, the pubis \& ischium. These differences in the osteolysis between the cemented and cementless cups does cause a more symptomatic loosening of the cemented cups allowing the patient to present early to the surgeon before substantial bone destruction had taken place. (Figure 7,8)

In contrast, the osteolysis in the cementless cups may present quite late as symptom may not have developed earlier. By that time a substantial expansile pattern would 


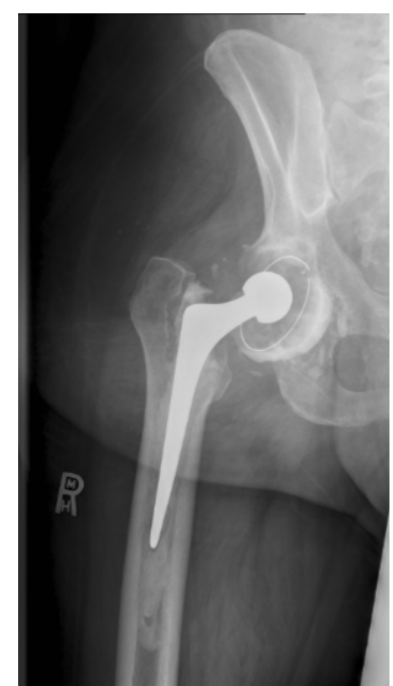

Figure 7. Osteolysis in cemented THA note the linear non expansile pattern
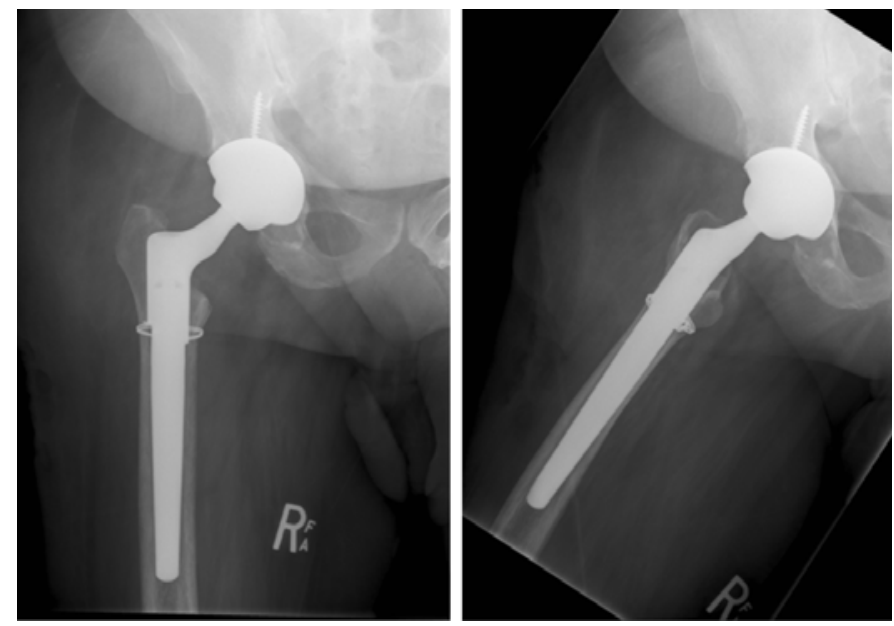

Figure 8. Post op , osteolysis in cemented THA note the simple revision of the cup, no defects.

have had developed with resultant bony defects. Occasionally it could be detected earlier on serial follow up with radiological diagnosis only in an asymptomatic or less symptomatic patient. [10,18] (Figure 7)

A classification system was proposed by Rubash, et al., for the uncemented cups based on the stability of the acetabular shell and the exchangeability of the liner. $[2,12,13]$

1. Type I, is the well fixed cup with focal osteolysis and the poly liner is exchangeable, in these cases if the cup position is acceptable it could be retained with change of the head an poly liner as well as debridement of the osteolytic defect.

2. Type II, is a well-fixed cup with focal osteolysis but the liner is not exchangeable. For example the locking mechanism is damaged, or the shell is worn out or malpositioned. In these cases the entire cup needs to be replaced.

3. Type III, is a loose cup requiring a complete revision.
The surgical choices that are available to the contemporary hip arthroplasty surgeons are basically one of two options. $[2,4,6,10,14]$ The first option lies in retaining the well fixed acetabular component. This certainly has it's own advantages in terms of being a surgery of lesser magnitude as well as a less financial burden, quicker rehabilitation and return to proper functional level in a short time span. It does, however, carry a risk of limited liner options based on the availability of the liners from the manufacturer if they still exist. The risk of instability has shown to be higher in the cases that were treated with liner change only.

The other option is to revise the cup completely, obviously this is a more extensive surgery with higher associated risks and complications. It does carry, in addition to all the risks of any revision surgery, a risk of possible incomplete Osseointegration, extensive bone stock damage, periprosthetic acetabular fracture or even pelvic discontinuity. But, on the other hand, it also has the advantage of using a larger cup with all the modern liner options, e.g, lateralized offset, harder bearing surfaces, and the ability to use larger head sizes to have a more stable reconstruct. It might be the only option in certain situations, e.g, infection, gross malposition, loose acetabular component, completely worn out and damaged acetabular shell. [2]

With both surgical interventions, addressing the osteolytic defect is of paramount importance, which involves aggressive surgical debridement and bone grafting using either autograft or allografts particulate material or bony substitutes. At the time of surgery if retention of the cup was undertaken, then the osteolytic defects could be accessed either through the screw holes or via trap door made in the ilium in the superoacetabular portion. [13] This is usually coupled with use of the more modern polyethylene liner that has a proven lower linear and volumetric wear rates, e.g, highly cross linked polyethylene liner. (Figure 9)

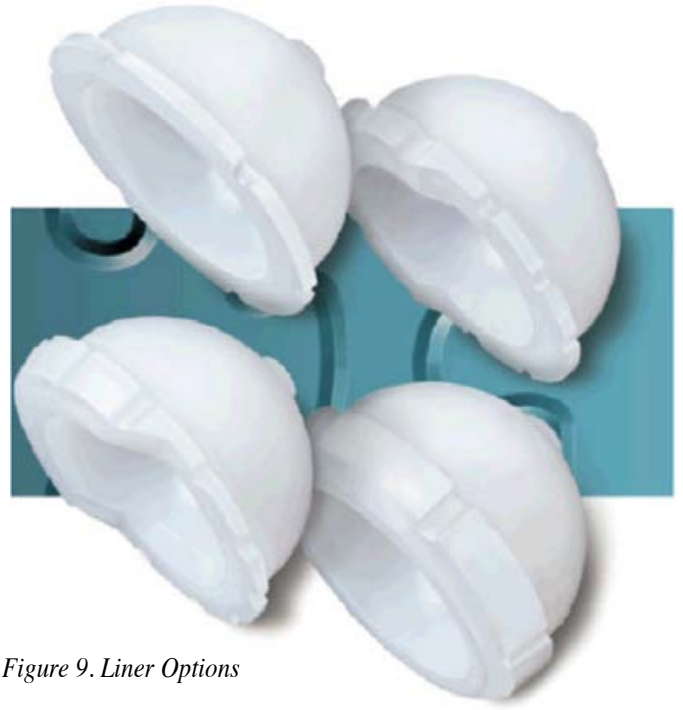


Preoperative assessment work starts with AP Pelvis \& Hip in supine position, with the hip in internal rotation, Judet views. [4] Assessing and quantifying osteolysis should be a critical part of the pre-operative planning and preparation of revision Total Hip Arthroplasty. The earliest hint of the presence of osteolysis should arise from the presence of discrepancy in position of femoral head within the hip joint in serial X-rays. This presence of asymmetric or symmetric wear should incite the surgeon to do a full extensive radiological workup looking for the presence or absence of osteolysis. Conventional X-rays should not be relied upon completely. They frequently miss some areas of osteolysis, a common area is the one posterior to the acetabular cup, which is also known (as the retroacetabular area) as well as that of the posterior wall. (Figure 4, 5)

Conventional X-rays are unlikely to pick the smaller osteolytic lesions. They always tend to underestimate the defect. [16] In terms of imaging modalities to detect and quantify the osteolytic lesion, magnetic resonance imaging (MRI) is considered to be the most sensitive at $95 \%$ and specific at $98 \%$, accuracy was found to be $96 \%$. [16] The sensitivity for detecting lesions by commuted tomography $(\mathrm{CT})$ scan is $75 \%$, while for plain X-rays is $52 \%$. [16] The location of the lesion did not affect the sensitivity of the MRI. However, the lesion size did correlate with the likelihood of its detection. Lesions of sizes greater than 3.0 cubic $\mathrm{cm}$ were not missed by the MRI. Although Computed tomography (CT) Scan did also detect lesions greater than $3.0 \mathrm{~cm} 3$ the MRI emerged to be the most effective study for detecting smaller size lesions, $<3.0 \mathrm{~cm} 3$. Unlike in MRI, and just like the conventional radiography, the location of the osteolytic lesion did influence the detection likelihood by the CT scan. The CT scan was more accurate in measuring the lesion volume compared to MRI.

Since most lesions of clinical concern are more than 3.0 $\mathrm{cm} 3$, both CT scan and MRI remain equally good for their detection rate. The choice of the appropriate modality by the treating surgeon remains a matter of cost control, the lesion location, and how symptomatic the patient is. If on plain radiograph an osteolytic lesion is seen and the patient is asymptomatic then further choice of imaging could be done as a preoperative planning tool rather than a diagnostic tool for detection of an osteolytic lesion.

Further preoperative workup in a patient with osteolysis who is being considered for surgery include a complete knowledge of the component(s) implanted, their manufacturer and the availability of compatible liners or any alternate ones that could be still applied to the cup.

The locking mechanism of the implanted acetabular cup needs to be investigated. In some instances the locking mechanism might have become defective or does not have a good enough track record. If the availability of a compatible liner cannot be guaranteed or the locking mechanism is defective, or it's efficiency is questionable, then alternative fixation methods, e.g, cement needs to be considered. $[2,14]$

The track record of the implanted cup is of paramount importance in preop decision making. For example, the modular ARTHROPOR cup (Joint medical products, Stamford, CT) has shown to be associated with delamination of its porous coating with $10.6 \%$ incidence. Although infrequent, delamination has also been reported for HarrisGalante cups. Knowledge of the mode of sterilization and shelf life of the liner will help in assessing the overall quality of the liner. [6]

Intraoperative assessment is the most accurate and reliable method to assess for loosening of the acetabular component. It has to be circumferentially exposed along with the bony edges to assess their relation to each other to enable the surgeon to determine if it's malpositioned or not. [2] The assessment of loosening is determined by surgeon critically examining all areas of bony ingrowth or on growth surfaces, as well as the presence of any tissues at the bone implant interface.

Pressure should be applied firmly through the central axis of the acetabular cup either manually or with the help of an acetabular pusher or inserter, or grasping the acetabular cup with a clamp through screw holes checking for any interface motion or expression of any fluid through the interface. Either of these findings do imply that the cup is loose and needs complete revision.

\section{Isolated Liner Exchange}

When this option is chosen by the surgeon several criteria should be met. The acetabular cup should be in an acceptable position and orientation to prevent instability. The compatible liners should be preferably available from the same manufacturer. Intraoperatively the surgeon may notice evidence of subtle malpositioning which he may try to correct by trialing various types of liners, e.g, lipped liner, lateralized (offset) liners, oblique liners, eccentric liners. If the instability cannot be corrected than the cup has to be completely revised. [2] (Figure 9) If the liners could be snapped in with the original intact locking mechanism that should be acceptable, alternatively the liner could be cemented into the preexisting well fixed cup.

The cementation of the liner into the acetabular component requires that the liner should be smaller (undersized) than the cup to allow for a minimum of $2 \mathrm{~mm}$ in thickness of the cement mantle. $[2,14]$ In cases of elderly patients 
with recurrent instability, who could be of high surgical risk, cementing a constraint liner remains a good salvage option. [2,14] The back of the liner and the inner aspect of the well fixed acetabular cup needs to be scored by a high speed burr to facilitate cement interdigitation. (Figure 10,11 )
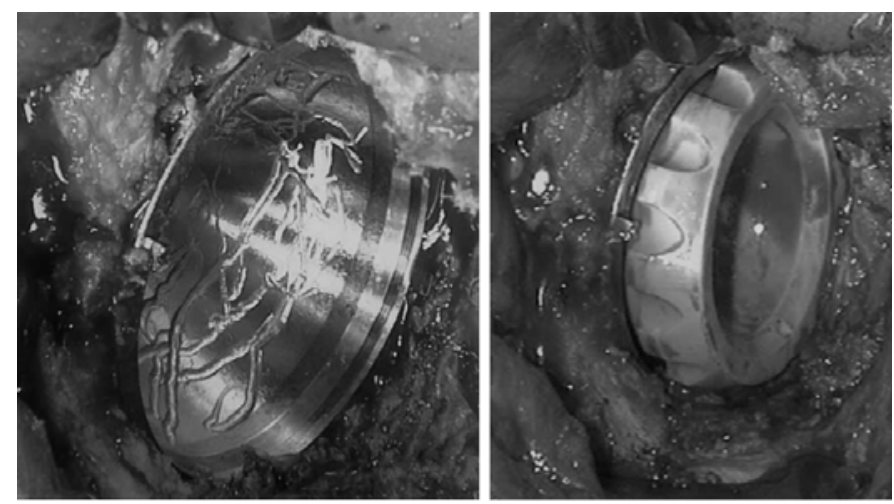

Figure 10. Cementing a Liner, scoring the retained cetabular shell
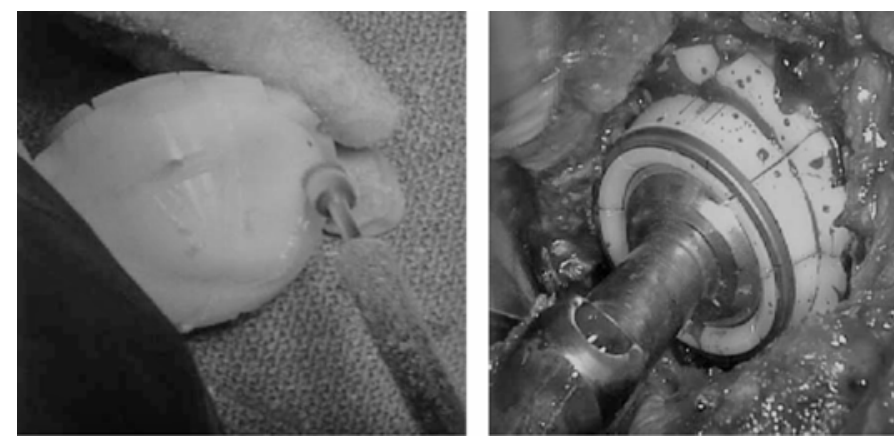

Figure 11. Cementing a Liner, scoring the back of the liner

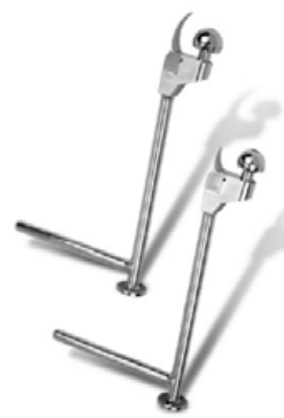

Figure 12. Explant Instrument for cup removal in revision THA

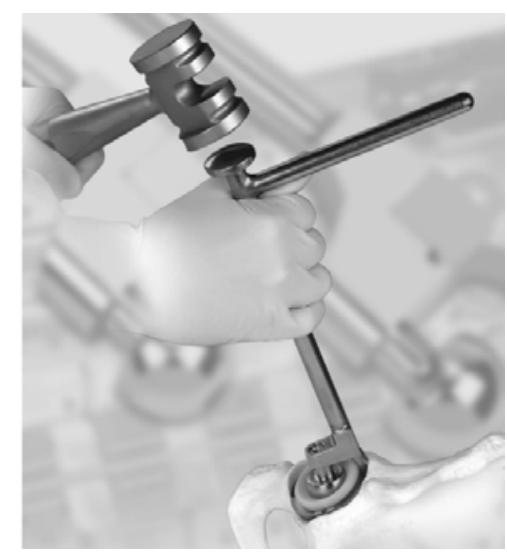

It needs to be emphasized that the commonest side effects that has been reported by various authors in the literature, is instability. Beaule, et al., reported $22 \%$ of dislocation in their series of 32 hips in which a liner was cemented in to a well-fixed socket. [1] Lie, et al., reported $28 \%$ incidence of dislocation in their study of a group of 1649 revision total hip arthroplasty from the Norwegian hip registry. [8] Their comparative groups of complete revision in patients with well-fixed cup and/or the patients with com- pletely loose cup had a lower chance of re revision. On the contrary, Blaha, reported on their group of 460 cases of revision THA, in 32 cases out of those, they decided to retain the liners, none of those 32 cases required further re revision. [2]

Few authors recommended using the direct lateral or anterolateral approaches for isolated liner change. O'Brien, et al., reported on their series of 24 THAs that were revised with isolated liner exchange with grafting of the defect, through direct lateral approach, they had no dislocations. [11] Wade, et al., reported on their series of 35 THAs that were revised with isolated polyethylene liner exchange, performed through anterolateral approach, there were 2 dislocations only (6\%). [15]

Boucher, et al., reported 25\% dislocation in their series of 24 THAs that were revised with isolated polyethylene exchange only through posterolateral approach. [3]

Complete revision of the acetabular component had become much technically easier with less potential of bone loss. The explant devices (Zimmer, Warsaw, IN), explant (Innomed, Savannah, GA), have facilitated the removal of acetabular cup without much damage to the host bone stock. (Figure 12)

\section{Conclusion}

In summary, polyethylene wear and osteolysis remains one of the most common causes of failure in contemporary THA. It remains the commonest cause of aseptic loosening in THA. Early identification of poly wear and osteolytic lesion with the option of exchanging the liner may be a useful technique to prevent a more catastrophic failure. Complete revision still remains an acceptable option in late cases, or in cases that the acetabular component is in an unacceptable position, infected, or damaged.

The surgeon should weigh the risks and benefits of both surgical options available to him and be fully prepared to alter the surgical course if the intraoperative findings did not support his original preoperative plan.

Even in cases of preoperatively planned isolated liner exchange only, full preparation must be undertaken to complete revision if it became evident that that's the appropriate choice to be made.

Due to the higher incidence of dislocation with isolated liner exchange, anti-instability measures should be adapted in terms of the surgical approaches, use of liners that decrease incidence of instability, post op rehab, and activity precaution. 


\section{References}

1. Beaulé, P. E., Ebramzadeh, E., Le Duff, M., Prasad, R., \& Amstutz, H. C. (2004). Cementing a liner into a stable cementless acetabular shell: the double-socket technique. The Journal of bone and joint surgery. American volume, 86-A(5), 929-934.

2. Blaha, J. D. (2002). Well-fixed acetabular component retention or replacement: the whys and the wherefores. The Journal of arthroplasty, 17(4 Suppl 1), 157-161.

3. Boucher, H. R., Lynch, C., Young, A. M., Engh, C. A., Engh, C., \& Black, J. (2003). Dislocation after polyethylene liner exchange in total hip arthroplasty. Journal of Arthroplasty, 18(5), 654-657.

4. Claus, A. M., Walde, T. A., Leung, S. B., Wolf, R. L., \& Engh, C. A. (2003). Management of patients with acetabular socket wear and pelvic osteolysis. The Journal of arthroplasty, 18(3 Suppl 1), 112-117.

5. Haft, G. F., Heiner, A. D., Callaghan, J. J., Dorr, L. D., Wan, Z., Long, W., Longjohn, D. B., et al. (2002). Polyethylene liner cementation into fixed acetabular shells. The Journal of arthroplasty, 17(4 Suppl 1), 167-170.

6. Hamilton, W. G., Hopper, R. H., Engh, C. Anderson, \& Engh, Charles A. (2010). Survivorship of polyethylene liner exchanges performed for the treatment of wear and osteolysis among porous-coated cups. Journal of Arthroplasty, 25(SUPPL. 6), $75-80$.

7. Hofmann, A. A., Prince, E. J., Drake, F. T., \& Hunt, K. J. (2009). Cementation of a Polyethylene Liner Into a Metal Acetabular Shell. A Biomechanical Study. Journal of Arthroplasty, 24(5), 775-782.

8. Lie, S. A., Hallan, G., Furnes, O., Havelin, L. I., \& Engesaeter, L. B. (2007). Isolated acetabular liner exchange compared with complete acetabular component revision in revision of primary uncemented acetabular components: a study of 1649 revisions from the Norwegian Arthroplasty Register. The Journal of bone and joint surgery. British volume, 89(5), 591-594.

9. Marshall, A., Ries, M. D., \& Paprosky, W. (2008). How prevalent are implant wear and osteolysis, and how has the scope of osteolysis changed since 2000? The Journal of the American Academy of Orthopaedic Surgeons, 16 Suppl 1, S1-S6.
10. Nayak, N. K., Mulliken, B., Rorabeck, C. H., Bourne, R. B., \& Robinson, E. J. (1996). Osteolysis in cemented versus cementless acetabular components. The Journal of arthroplasty.

11. O'Brien, J. J., Burnett, R. S. J., McCalden, R. W., MacDonald, S. J., Bourne, R. B., \& Rorabeck, C. H. (2004). Isolated liner exchange in revision total hip arthroplasty. The Journal of Arthroplasty.

12. Rubash HE, Sinha RK, Engh CA, et al: A new classification system for the management of acetabular osteolysis after total hip arthroplasty. Instr Course Lect (48):37, 1999

13. Saleh, K., Thongtrangan, I., Schwarz, E. (2004). Osteolysis, Medical and Surgical Approaches. Clinical Orthopedics and Related Research. Vol.427, pp.138-147.

14. Springer, B. D., Hanssen, A. D., \& Lewallen, D. G. (2003). Cementation of an acetabular liner into a well-fixed acetabular shell during revision total hip arthroplasty. Journal of Arthroplasty (Vol. 18, pp. 126-130).

15. Wade, F. A., Rapuri, V. R., Parvizi, J., \& Hozack, W. J. (2004). Isolated acetabular polyethylene exchange through the anterolateral approach. Journal of Arthroplasty, 19(4), 498-500.

16. Walde TA, Weiland DE, Leung SB, et al. Comparison of CT, MRI, and radiographs in assessing pelvic osteolysis: a cadaveric study. Clin Orthop Relat Res. 2005; (437):138-144.

17. Zhu, Y. H., Chiu, K. Y., \& Tang, W. M. (2001). Review Article: Polyethylene wear and osteolysis in total hip arthroplasty. Journal of orthopaedic surgery (Hong Kong), 9(1), 91-99. Retrieved from http://www.ncbi.nlm.nih.gov/ pubmed/12468851

18. Zicat, B., Engh, C.E., (1995). Patterns of Osteolysis Inserted with around Total Hip Components and without. J. Bone Joint Surg. Am, 77-A(3), 432-439.

\section{Copyright \& Licensing}

Authors retain copyright and grant the journal right of first publication with the work. Reconstructive Review follows the Creative Commons Attribution-NonCommercial CC BY-NC. This license allows anyone to download works, build upon the material, and share them with others for non-commercial purposes as long as they credit the senior author, Reconstructive Review, and the Joint Implant Surgery \& Research Foundation (JISRF). An example credit would be: "Courtesy of (senior author's name), Reconstructive Review, JISRF, Chagrin Falls, Ohio".

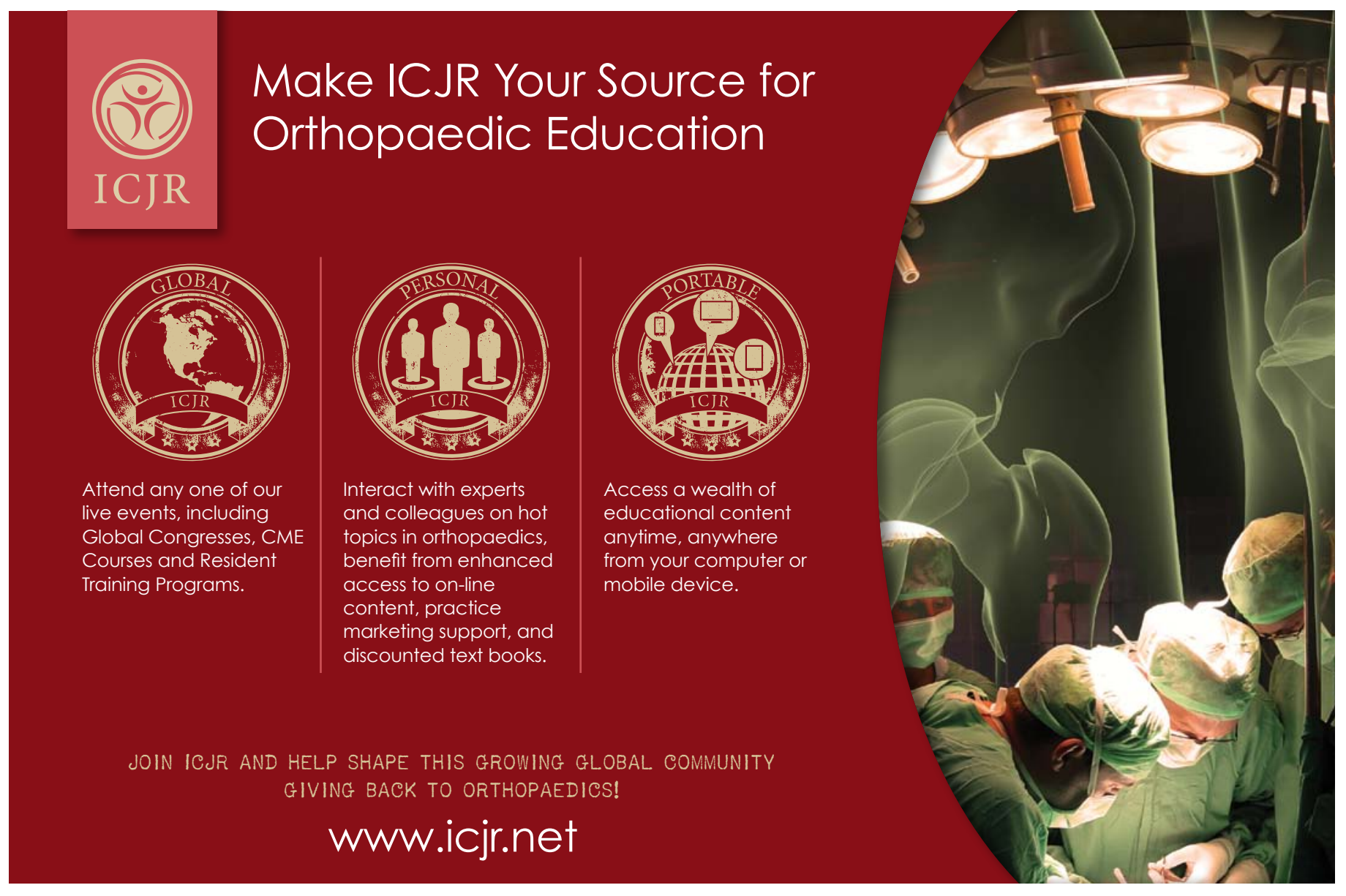

